\title{
Latest Developments in Cellular Therapy for Multiple Myeloma
}

\author{
Tara K Gregory, ${ }^{1}$ Henning Schade, ${ }^{1}$ and Jesús G Berdeja ${ }^{2}$ \\ 1. Sarah Cannon Research Institute at the Colorado Blood Cancer Institute, Denver, CO, USA; 2. Sarah Cannon Research Institute, Nashville, TN, USA
}

DOI: https://doi.org/10.17925/OHR.2021.16.2.111

$\mathrm{N}$ ovel drugs and continuous therapy have improved outcomes in patients with multiple myeloma (MM); however, current therapies are largely non-curative with diminishing durations of response with subsequent therapies. Cellular therapies including chimeric antigen receptor T (CAR-T) cells have started to illustrate deeper and longer durations of relapse in the relapsed/refractory population. Current research seeks to expand on the durability of response, novel combinations, and targets, as well as the timing of CAR-T cells in the treatment schema. This review summarizes the updates in cellular therapy for MM.

\section{Keywords}

Multiple myeloma, CAR-T, chimeric antigen receptor T cells, BCMA, B cell maturation antigen

Disclosures: Sarah Cannon Research Institute receives research funds in Tara K Gregory's name for the following: Abbvie, Amgen, Acetylon, Bluebird, Bristol-Myers Squibb, Celgene, Celularity, Constellation, CRISP Therapeutics, CURIS, EMD Sorono, Genentech, Glenmark, Janssen, Kesios, Lilly, Novartis, Poseida, Sanofi, Takeda, Teva, and Vivolux. Sarah Cannon Research Institute receives research funds in Henning Schade's name for the following: Abbvie, Amgen, Acetylon, Bluebird, BristolMyers Squibb, Celgene, Cellularity, Constellation, CRISP Therapeutics, CURIS, EMD Sorono, Genentech, Glenmark Janssen, Kesios, Lilly, Novartis, Poseida, Sanofi, Takeda, and Vivolux. Sarah Cannon Research Institute receives research funds in Jesús $\mathrm{G}$ Berdeja's name for the following: Abbvie, Amgen, Acetylon, Bluebird, Brsitol-Myers Squibb, Celgene, Cellularity, Constellation, CRISP Therapeutics, CURIS, EMD Sorono, Genentech, Glenmark, Janssen, Kesios, Lilly, Novartis, Poseida, Sanofi, Takeda, and Vivolux.

Review Process: Double-blind peer review.

Compliance with Ethics: This article involves a review of multiple myeloma CAR-T cell therapy via a PubMed literature review, recent presentations at medical conferences, and ongoing research at ClinicalTrials. gov. This article did not involve any studies with human or animal subjects performed by any of the authors.

Authorship: The named authors meet the International Committee of Medical Journal Editors (ICMJE) criteria for authorship of this manuscript, take responsibility for the integrity of the work as a whole, and have given final approval for the version to be published.

Access: This article is freely accessible at touchONCOLOGY.com (c) Touch Medical Media 2021.

Received: July 9, 2020

Accepted: December 21, 2020

Published Online: February 8, 2021

Citation: Oncology \& Hematology Review. 2020; 16(2):111-8

Corresponding Author: Tara K Gregory, Colorado Blood Cancer Institute, 1721 East 19th Avenue, Suite 300, Denver, Colorado 80218. E: Tara.Gregory@HealthONEcares.com Twitter: @TaraGregoryMD

Support: No funding was received in the publication of this article.
Multiple myeloma (MM) is a malignant clonal disorder of plasma cells in the bone marrow, and more than 32,000 new cases are expected in the USA each year. ${ }^{1}$ Many clinical advances, including novel drugs and continuous therapy with treatment sequencing, have improved survival statistics. ${ }^{2}$ Despite this, MM remains largely incurable and the relapsed/refractory population remains vulnerable despite numerous new therapeutic options. ${ }^{3.4}$ Chimeric antigen receptor T (CAR-T) cell therapy offers early impressive results in the relapsed/refractory setting, providing hope that these therapies and others like it may be heralding in a new era in the treatment of $\mathrm{MM}$, which may continue to move us closer to that elusive cure. CAR-T cell studies focus on the heavily pretreated patient with MM, and future studies that focus on its placement in the treatment sequence as well as optimum targets.

\section{Background on CAR-T cells}

CAR-T cell therapy combines optimal antigen binding of a tumor cell surface molecule and direct T-cell activation inciting a targeted immune response as a novel class of "living" drug in malignancies. $T$ cells are collected via apheresis and then genetically engineered to express an artificial receptor or CAR. The CAR consists of an extracellular antigen recognition domain or targeting domain, a transmembrane domain, and an intracellular domain to activate effector functions in the T cell. ${ }^{5}$ Second- and third-generation CARs combine additional co-stimulatory ligands, such as CD28 or 4-1BB signaling domains, to induce cytokine production and enhance function, differentiation, and persistence of the adoptive T cell (Figure 1). ${ }^{6}$

Multiple investigators concurrently working on cell therapy described a murine B-cell malignancy model in which T cells were transduced via a retroviral mechanism to target CD19.? Treatment moved on to human subjects with acute lymphoblastic leukemia (ALL) and advanced B-cell malignancies. A phase II study using the anti-CD19 CAR-T product tisagenlecleucel in pediatric and young adult patients with CD19+ relapsed. Refractory B-cell ALL resulted in an overall remission at 3 months of $81 \%$ leading to the product's US Food and Drug Administration (FDA) approval in August 2017.8 In advanced B-cell malignancies, a lympho-depleting conditioning regimen with fludarabine and cyclophosphamide followed by adoptive T-cell transfer in 22 patients showed an overall response rate (ORR) of 75\% including 55\% complete remission (CR) and $18 \%$ partial remission (PR). ${ }^{9}$ This was quickly followed with the FDA approval of axicabtagene ciloleucel in October 2017 after a phase II trial in 111 patients showed a 99\% manufacturing success rate and an ORR of $82 \% .^{10}$

\section{Multiple myeloma B-cell maturation antigen CAR-T target}

Anti-CD19 CAR-T cell has been explored in MM in addition to aggressive B-cell malignancies. Although MM cells express CD19 infrequently, CTL019 cells are cytotoxic at low levels of CD19 expression. At the University of Pennsylvania, Philadelphia, PA, USA, a compassionate use pilot study of CTL019 cells were infused day 12 after high-dose melphalan and autologous stem cell infusion. The patient did not develop cytokine release syndrome (CRS) and obtained a CR. 10,11 Despite this proof of concept, the majority of MM cells do not express CD19. 


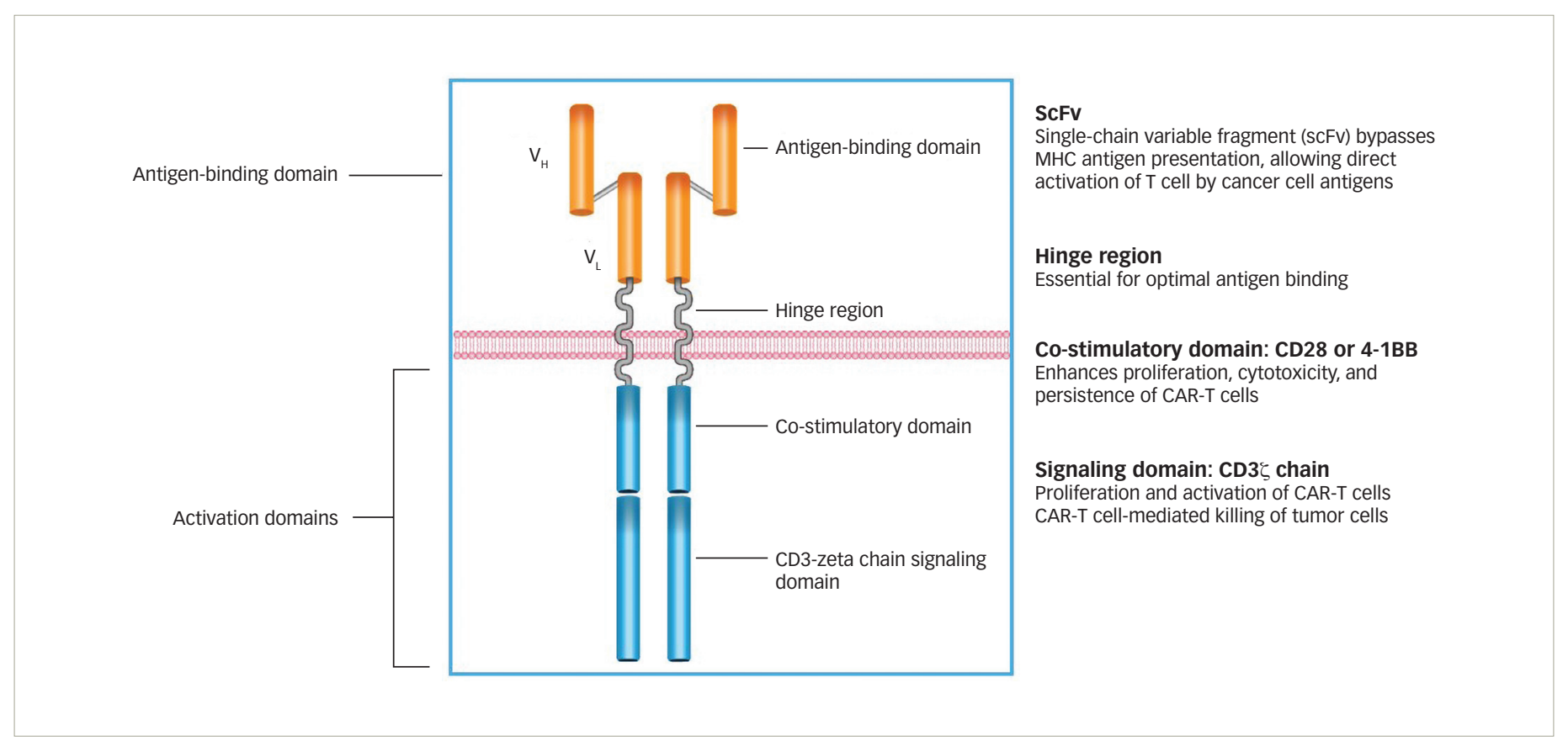

$C A R=$ chimeric antigen receptor; $M H C=$ major histocompatibility complex

Since then there has been an explosion of CAR-T cell therapies in MM. $B$ cell maturation antigen (BCMA) has been the most important target to date. ${ }^{12}$ BCMA-a type III transmembrane protein-is universally expressed on the surface of malignant plasma cells and a small fraction of mature B cells; however, it is not expressed on normal human tissue, including the primary human $\mathrm{CD} 34+$ hematopoietic cell. As a member of the tumor necrosis factor superfamily, its overexpression augments MM cell growth and survival serving as a receptor for B-cell activating factor (BAFF) and a proliferation-inducing ligand (APRIL). ${ }^{13,14}$ BCMA maintains surface and intracellular expression through relapse, extramedullary spread, and residual disease after treatment, making it an attractive therapeutic target. ${ }^{15}$ Preclinical results of anti-BCMA CARs transduced with lentiviral vectors led to the specific killing of two-thirds of autologous primary MM cells leading to the initial in vivo studies. ${ }^{16}$ Other targets such as GPRC5D, SLAMF7, and CD138 are being developed as well, but are well behind BCMA.

\section{First wave CAR-T cells for multiple myeloma} Initial clinical data

The first clinical data of a BCMA CAR-T cells included 12 patients with a median of seven prior treatments with an anti-BMCA CAR containing a murine single-chain variable fragment (SCFV), a CD8 $\alpha$ hinge and transmembrane region, a CD28 co-stimulatory domain, and a CD3 $\zeta$ signaling domain. Patients were dosed from $0.3 \times 10^{6}$ CAR-T cells/kg up to $9 \times 10^{6}$ CAR-T cells $/ \mathrm{kg}$. This resulted in seven patients who achieved stable disease (SD): three with PR, one with very good partial remission (VGPR), and one with stringent complete remission (SCR). The two patients treated with the highest dose experienced complete and rapid elimination of bone marrow involvement from MM. ${ }^{17}$ In total, 26 patients were enrolled in this study with 16 at the highest dose level of $9 \times 10^{\circ} / \mathrm{kg}$ preceded by a conditioning regimen of cyclophosphamide and fludarabine. ${ }^{18}$ An impressive ORR of $81 \%$ was reported, and high peak blood CAR-T cell levels were reportedly associated with response. Importantly, all 11 patients who obtained remission greater than PR achieved minimal residual disease (MRD) negative status by flow cytometry. CRS, with hypotension, hypoxemia, fevers, and tachycardia, as well as coagulopathy and neurotoxicity with delirium, was described with the highest doses, which is similar to what has been seen in previous antiCD19 CAR-T cell trials. Six of the 16 patients required vasopressors, but CRS was reversible in all cases. These positive results provided the foundation of future BCMA-directed CAR-T cell trials (Figure 2).

Ide-cel

Ide-cel (idecabtagene vicleucel, bb2121; Bristol-Myers Squibb, New York, NY, USA and bluebird bio, Inc., Cambridge, MA, USA) is a second-generation CAR-T cell product based on the initial product utilized by the Kochenderfer team. It is an autologous T-cell product transduced with a lentiviral vector encoding an anti-BCMA single-chain variable fragment, a CD137 (4-1BB) co-stimulatory domain, and a CD3- $\zeta$ signaling domain (Table 1). In the phase I CRB-401 study, ide-cel was infused using doses of $50 \times 10^{6}$ up to $800 \times 106$ CAR+ T cells. Responses were only seen at the $150 \times 10^{6}$ dose and higher, leading to $150 \times 10^{6}$ up to $450 \times 10^{6} \mathrm{CAR}+\mathrm{T}$ cells in the expansion phase. The 33 enrolled patients were heavily pretreated with a median of seven lines of prior therapies. The most common $\geq$ grade 3 toxicities were neutropenia (85\%), leukopenia (58\%), anemia (45\%), and thrombocytopenia (45\%). CRS was reported in $76 \%$ of patients, but were mostly grade 1 or 2 , and only $6 \%$ were $\geq$ grade 3 . Neurotoxicity occurred in $42 \%$ of patients, and were mostly grade 1 or 2 ; only one patient (3\%) had a reversible grade 4 neurotoxicity. The ORR was $85 \%$, including $45 \%$ with complete responses - $40 \%$ of whom subsequently relapsed. The median progression-free survival (PFS) was 11.8 months (95\% confidence interval: 6.2-17.8). All patients who responded with $\geq P R$ were evaluated for MRD-negative status ( $\leq 10-4$ nucleated cells). Ide-cel expansion correlated with responses; interestingly, the responses were not BCMA-expression dependent. CAR-T cells subsisted up to 1 year after treatment. ${ }^{19}$ 
Figure 2: Multiple myeloma and CAR-T cell studies—timeline of earliest and most advanced products

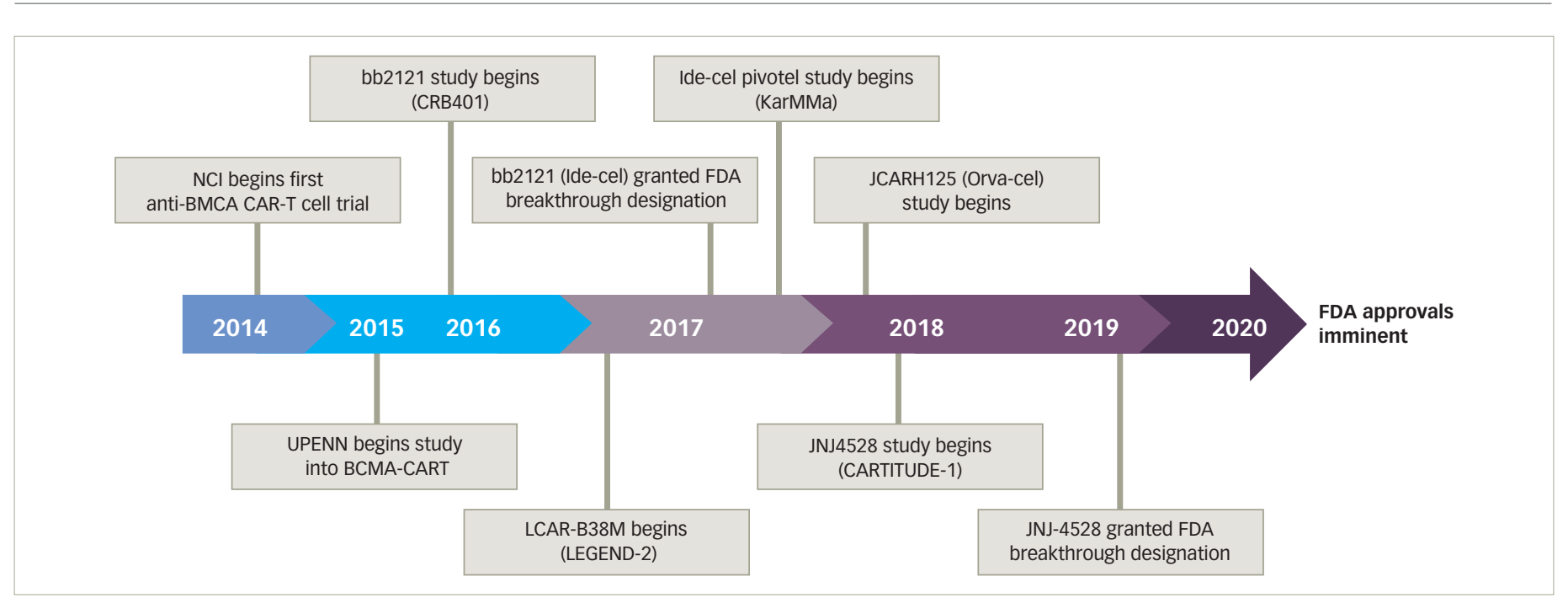

$B M C A=B$-cell maturation antigen; CAR = chimeric antigen receptor; FDA = US Food and Drug Administration; NCI = National Cancer Institute; UPENN = University of Pennsylvannia, $P A, U S A$.

Table 1: Characteristics of leading BCMA-directed CAR-T cells in multiple myeloma

\begin{tabular}{|c|c|c|c|}
\hline & Ide-cel ${ }^{20}$ & Orva-cel ${ }^{34}$ & Cilta-cel ${ }^{26}$ \\
\hline Nomenclature & $\begin{array}{l}\text { bb2121 } \\
\text { Idecabtagene vicleucel }\end{array}$ & $\begin{array}{l}\text { JCARH125 } \\
\text { Orvacabtagene autoleucel }\end{array}$ & $\begin{array}{l}\text { LCAR-B38M } \\
\text { JNJ-68284528 }\end{array}$ \\
\hline Target & BCMA & BCMA & BCMA \\
\hline Ag-binding domain & SCFV (M) & SCFV (H) & 2-VHH (C) \\
\hline Vector & Lentiviral & Lentiviral & Lentiviral \\
\hline Co-stimulatory domain & $\mathrm{CD} 3 / 41 \mathrm{BB}$ & $\mathrm{CD} 3 / 41 \mathrm{BB}$ & $\mathrm{CD} 3 / 41 \mathrm{BB}$ \\
\hline Special qualities & & $\begin{array}{l}\text { Modified spacer to enhance binding } \\
\text { Designed to deliver purified CD4+ and CD8+ CAR-T cell product enriched for } \\
\text { central memory phenotype }\end{array}$ & $\begin{array}{l}\text { Two BCMA binding domains to } \\
\text { enhance avidity }\end{array}$ \\
\hline
\end{tabular}

$A g=$ antigen; $B C M A=B$ cell maturation antigen; $C=$ camelid; $C A R T=$ chimeric antigen receptor $T$ cells; $H=$ human; $M=$ murine; scFv = single chain variable fragment; $V H H=$ variable heavy chain.

These findings lead to the confirmatory pivotal phase II KarMMa trial of ide-cel for patients with relapsed/refractory MM (RRMM) (Table 2). ${ }^{20}$ Patients who had $\geq 3$ prior regimens (including immunomodulatory drug (IMiD), proteasome inhibitor (PI), and CD38 monoclonal antibody), and who were refractory to their last regimen as per International Myeloma Working Group (IMWG) criteria, were enrolled. After conditioning with cyclophosphamide and fludarabine, $150-450 \times 106$ CAR+ T cells were given to each patient. A total of 128 patients with a median age of 61 years received a median of six prior regimens; $84 \%$ were triple-refractory and $26 \%$ were penta-refractory. At a median follow up of 11.3 months, the ORR was $73 \%$ including $33 \% \mathrm{CR} / \mathrm{SCR}$, with a median PFS of 8.6 months. The responses appear to be dose dependent; the $150 \times 10^{6}$ dose induced 50\% ORR with each 25\% CR and VGPR; the median duration of response (DOR) was not reached and the PFS was 2.8 months. The $300 \times 10^{6}$ dose appeared to be more efficient with an ORR of $69 \%$ including $29 \%$ CR, $14 \%$ VGPR and $26 \%$ PR; the median DOR was 9.9 months, and the PFS was 5.8 months. The $450 \times$ $10^{6}$ dose appeared to be the most efficacious with an ORR of $82 \%$ including $39 \%$ with CR/SCR, $26 \%$ with VGPR, and $17 \%$ with PR; the median DOR was 11.3 months and the PFS was 12.1 months. The median overall survival (OS) was impressive with 19.4 months for all patients and all subgroups, including older and high-risk patients, who appeared to benefit equally.
In 33 patients who achieved CR/SCR, 79\% were MRD-negative by nextgeneration sequencing (NGS) at a cutoff of $10^{-5}$ nucleated cells. Cytopenias (97\%) and CRS (84\%) were common, although CRS was mainly grade 1-2. The median onset to CRS was 1 day and only seven patients had grade $3-5$ CRS. Neurotoxicity developed in 18\%, but only $3 \%$ had grade 3 and no grade $\geq 4$ were reported. A median peak CAR $+\mathrm{T}$ cell expansion occurred at 11 days, and the expansion of CAR-T cells was higher in responders. CAR-T cells were detected in $59 \%$ of patients at 6 months and in $36 \%$ at 12 months post-treatment. An additional cohort enrolled three patients at an $800 \times 10^{6}$ dose with an ORR of $100 \%$, including $100 \%$ with CR and MRD negative. ${ }^{21}$

\section{Cilta-cel}

LCAR-B38M is an autologous CAR-T cell product that also contains a 4-1BB co-stimulatory domain and a CD3- $\zeta$ T-cell signaling domain, and two BCMAtargeting single chain antibodies (Table 1). LEGEND-2 is a first-in-human, phase I, single arm, clinical trial conducted in RRMM performed at four centers in China using the LCAR-B38M construct. ${ }^{22}$ The study enrolled 74 patients at four sites with 57 patients enrolling from one study site. These 57 patients had a median of three prior lines of therapy; 68\% were PI exposed, $86 \%$ IMiD exposed, and $60 \%$ had both prior PI and IMiD exposure. Few were daratumumab exposed. Patients received single-agent cyclophosphamide 
Table 2: Summary of completed and ongoing pivotal BCMA-directed CAR T trials in multiple myeloma including the products Ide-cel, Orva-cel, and Cilta-cel

\begin{tabular}{|c|c|c|c|}
\hline & $\mid d e-$ cel $^{20}$ & Orva-ce| $^{34}$ & Cilta-cel ${ }^{26}$ \\
\hline Study & KarMMa & EVOLVE & CARTITUDE-1 \\
\hline Number of patients treated & 128 & 62 & 97 \\
\hline Population & Relapsed/refractory & Relapsed/refractory & Relapsed/refractory \\
\hline Number of prior treatments & 6 & 6 & 6 \\
\hline CAR $+T$ cell dose & $150-450 \times 10^{6}$ & $300-600 \times 10^{6}$ & $0.75 \times 10^{6} / \mathrm{kg}$ \\
\hline ORR & $73 \%\left(82 \%\right.$ at $\left.450 \times 10^{6}\right)$ & $92 \%$ & $94.8 \%$ \\
\hline CR & $33 \%\left(39 \%\right.$ at $\left.450 \times 10^{6}\right)$ & $36 \%$ & $67 \%$ \\
\hline $\begin{array}{l}\text { MRD negative } \\
(\geq 10-5 \text { by NGS) }\end{array}$ & $\begin{array}{l}79 \% \\
\text { (33 patients in } \geq \text { CR) }\end{array}$ & $\begin{array}{l}84 \% \\
(\geq \mathrm{PR} \text { at month } 3)\end{array}$ & $\begin{array}{l}94 \% \\
\text { (of } 52 \text { patients evaluable) }\end{array}$ \\
\hline $\begin{array}{l}\text { CRS } \\
\text { All grades (grade 3/4) }\end{array}$ & $84 \%(5 \%)$ & $89 \%(3 \%)$ & $92 \%(5 \%)$ \\
\hline $\begin{array}{l}\text { Neurotoxicity } \\
\text { All grades (grade 3/4) }\end{array}$ & $18 \%(3 \%)$ & $13 \%(3 \%)$ & $20.6 \%$ (2.1\%) \\
\hline Median time to CRS & 1 day & 2 days & 7 days \\
\hline Median PFS & $\begin{array}{l}8.8 \text { months } \\
\left(12.1 \text { months at } 450 \times 10^{6}\right)\end{array}$ & Not reported & $76.6 \%$ at 12 months \\
\hline Median OS & 19.4 months & Not reported & Not reported \\
\hline CAR-T persistence & $\begin{array}{l}59 \% \text { at } 6 \text { months } \\
36 \% \text { at } 12 \text { months }\end{array}$ & $69 \%$ at 6 months & $67 \%$ at 6 months \\
\hline
\end{tabular}

$B C M A=B$-cell maturation antigen; $C A R=$ chimeric antigen receptor; $C R=$ complete response; $C R S=$ cytokine release syndrome; $M R D=$ minimal residual disease; $N G S=$ next generation sequencing; $O R R=$ overall response rate; $O S=$ overall survival; $P F S=$ progression-free survival; $P R=$ partial remission .

followed by three sequential infusions with LCAR-B38M at 20\%, 30\%, and $50 \%$ of the total dose. Common adverse events (AEs) reported were fever (91\%), CRS (90\%), thrombocytopenia (49\%), and leukopenia (47\%). Grade $\geq 3$ AEs were reported in two-thirds of patients, specifically leukopenia (30\%), thrombocytopenia (23\%), and increased aspartate aminotransferase (21\%). CRS occurred in $90 \%$ of patients and was primarily grade $1-2(82 \%)$; only four patients (7\%) had grade 3 events, and no grade 4-5 CRS was observed. Neurotoxicity was observed in only one patient (grade 1 aphasia, agitation, seizure-like activity). The median time to onset of CRS was 9 days, and all but one CRS events resolved. The ORR was $88 \%$, and CR was $74 \%$. Impressively, median PFS was 19.9 months and 28.2 months, respectively, for patients who achieved CR. ${ }^{23}$

To confirm these results from LEGEND-2 in a more homogenous relapsed/ refractory population, the phase IB/II dose-confirming study CARTITUTE-1 was initiated (Table 2). Cilta-cel (ciltacabtagene autoleucel, JNJ-4528, LCAR-B38M/JNJ-68284528; Janssen Pharmaceuticals, Beerse, Belgium and Legend Biotech Somerset, Ewing Township, NJ, USA) is identical to the CAR construct used in the LEGEND-2 study. ${ }^{24}$ In the phase $\mathrm{Ib}$ portion of the study, 29 patients received cyclophosphamide and fludarabine lymphodepletion and then a single infusion at a target dose of $0.75 \times 10^{\%} /$ $\mathrm{kg}$. Patients were heavily pretreated with a median of five prior lines of therapy. Median time to CRS was 7 days, which is different when compared with the median onset of CRS from Ide-cel, which is only one day. Only 10\% of patients developed neurotoxicity, and only $3 \%$ were $\geq$ grade 3 . The ORR was $100 \%$, the SCR $86 \%$, the VGPR $\geq 97 \%$, and the PR $3 \%$. The median time to SCR was 3 months, and the 9-month PFS was $86 \%$. Of the 16 patients in CR, 13 were MRD-negative by NGS at a cutoff of $10^{-5}$ nucleated cells, and 11 at a cutoff of $10^{-6}$ nucleated cells. Interestingly, at the 6-month follow up, 22 of 28 patients had CAR+ T cells below the level of quantification in peripheral blood, suggesting that CAR-T persistence may not correlate with the depth of response to Cilta-cel..$^{25}$ Updated phase Ib/II follow-up data in 97 patients with a 12.4-month follow up showed an ORR of $96.9 \%$, and a CR of $67 \%$. Median PFS was not reached and low-grade CRS occurred in $92 \%$ of patients, still with a later onset of 7 days. New neurotoxicity was $20.6 \%$ anygrade neurotoxicity, and $10.3 \%$ were grade 3 or greater. This was further divided into immune effector cell-associated neurotoxicity syndrome (ICANS) or other neurotoxicities, such as neurocognitive changes, nerve palsy, and peripheral motor neuropathy. ICANS of all grades occurred in $16.5 \%$ of cases, and $\geq$ grade 3 in $2.1 \%$, with a median time to onset of 8 days and median duration of 4 days. Other neurotoxicities of any grade occurred in $12.4 \%$ of patients, and $\geq$ grade 3 occurred in $9.3 \%$, with a late median time-to-onset of 27 days and a duration of 75 days. ${ }^{26}$ Further phase II outcomes are eagerly awaited.

\section{Mechanisms of resistance to B-cell maturation antigen CAR-T cell therapy}

Despite impressive response rates and durability in RRMM patients, the majority will eventually relapse. The mechanisms of resistance to BCMAdirected CAR-T cell therapy are not well understood. ${ }^{27}$ Unlike CD19, where up to $75 \%$ of relapsed patients had loss of expression of the CD19 antigen

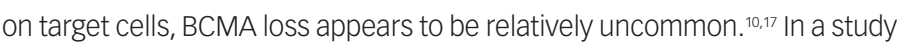
by Ali et al., a partial loss of BCMA expression by malignant plasma cells was detected only in 1 of 12 patients. In the KarMMa trial only two patients who progressed were found to have lost BCMA expression on myeloma cells. ${ }^{17}$ This suggests that patients who progressed after BCMA-targeted therapy may very well respond to alternate BCMA-targeted therapy. ${ }^{28}$ it is imperative that these patients are not excluded from clinical trials directed at BCMA as is the current practice. Relapse appears to be occurring both in the setting of loss of CAR-T cells and in the setting of persistence of CAR-T 
cells. Thus various mechanisms appear to be at play including anti-BMCA CAR-directed antibodies and off-target binding of BCMA CARs on soluble BCMA (SBCMA). Additionally, reversible antigen loss through trogocytosis can occur by transferring the target antigen to $T$ cells. This antigen loss leads to a decrease of target density on tumor cells, and inhibits T-cell activity via T-cell exhaustion or even T-cell killing. Furthermore, T-cell exhaustion with chronic antigen exposure, overexpression of programmed cell death protein-1 (PD-1) post infusion leading to functional BCMA CAR-T cell inhibition via PD-1/programmed death-ligand 1 (PD-L1) axis, and upregulation of tumor inhibitory signals have been postulated. . $^{17,29-32}$ As with prior therapies in $\mathrm{MM}$, a combination approach seems to be necessary to best control the disease. Could the intrinsic heterogeneity of $\mathrm{MM}$ require that this same paradigm be followed with CAR-T cell therapy? Perhaps combinations with various immune-modulating therapies, such as IMiDs and anti-CD38 antibodies, may ultimately be required. This is also an opportunity where dual targeting CAR-T cell products may prove advantageous.

\section{Second-wave CAR-T cells for multiple myeloma}

Several new-generation products are now being tested clinically, building on the impressive results of the earlier products with the aim of overcoming some of the possible resistance mechanisms. The efficacy of gene transfer makes it feasible to express CARs in specific subsets of T cells. Central memory (Tscm) cells have superior proliferative capacity, Ionger telomeres, and improved survival after adoptive transfer compared to effector memory (TEM) and effector (TE) T cells..$^{33}$ Thus, efforts to select for this T-cell phenotype has been a priority goal of the next generation of products.

\section{Orva-cel}

Orva-cel (orvacabtagene autoleucel, Juno Therapeutics, Seattle, WA, USA) is an anti-BMCA CAR containing a lentiviral construct with a fully human SCFV, an optimized spacer, and 4-1BB co-stimulatory and CD3- $\zeta$ activation domains (Table 1). It avoids off-target binding with a Iow affinity for SBCMA, is active on target cells that express Iow BCMA density, minimizes tonic signaling to reduce antigen-independent exhaustion, and the modified spacer increases engagement of the antigen binding to the SCFV, thus increasing cell kill. ${ }^{34}$ It is manufactured to produce an equal CD4:CD8 ratio enriched for a central memory phenotype thought to increase persistence and durability. The phase I/ II EVOLVE trial enrolled 62 patients with RRMM who previously received a median of six prior lines of therapy. The trial included five dose level escalations of $50 \times 10^{6}, 150 \times 10^{6}, 300 \times 10^{6}, 450 \times 10^{6}$, and $600 \times 10^{6}$ (Table 2). At the three highest dose levels, cytopenias were more frequent. Two deaths occurred due to infection and grade 5 mast cell activation syndrome (MAS)/hemophagocytic lymphohistiocytosis (HLH). Overall, CR occurred in $89 \%$ of patients with only two experiencing $\geq$ grade 3 events, and had a median time-to-onset of 2 days, and a median timeto-resolution of 4 days. At a median follow up of 6.9 months, the ORR was $92 \%$, with CR of $36 \%$, VGPR of $32 \%$, and PR of $24 \%$. At the $600 \times 10^{6}$ dose level, $100 \%$ of patients were MRD-negative at $10^{-5}$ nucleated cells by NGS at 3 months. The trial has a 100\% manufacturing success rate with robust expansion at all dose levels through day 29 with a trend towards increased expansion at greater dose levels, and 69\% CAR persistence at 6 months. High baseline levels of sBCMA did not impact orva-cel activity. Based on these data, the phase II study plans to move forward at the $600 \times 10^{6}$ dose level. ${ }^{35}$

\section{bb21217}

bb21217 is identical to ide-cel (bb2121) utilizing the same ScFV, 4-1BB co-stimulatory domain and CD3- $\zeta$ activation domains with the exception of co-culturing with a phosphoinositide 3 kinase inhibitor (PI3K) bb007 during ex vivo culture to enrich the drug product for T cells displaying a memory-like phenotype. In preclinical models, both CARs eliminated MM tumors in mouse models, but the bb21217 product was able to prevent tumor growth when re-challenged with a second tumor without reinfusion of cells. The bb21217 CAR showed higher levels of CCR7 and CD27, suggesting a higher level of memory-like T cells and lower levels of CD57, which is a marker of T cell exhaustion..$^{36,37}$ In the phase I study, dose levels include $150 \times 10^{6}$ $(n=12), 300 \times 10^{6}(n=14)$, and $450 \times 10^{6}(n=43)$. At a 23-month follow up of the first dosing cohort, the ORR is $83 \%$ and the median duration of response is 11.1 months. ${ }^{38} \mathrm{~A}$ proprietary manufacturing change took place at dose level 3. The original ORR was $60 \%$ with a CR of $32 \%$. In the modified dose level 3 cohort, the ORR was $84 \%$ with a CR of $32 \%$. All patients in CR were also MRD-negative, and the duration of response in this cohort at the 7-month follow up was not reached. Increased Tscm expansion correlated with the increase in level and the duration of response..$^{39}$ In subsequent follow ups, it will be interesting if apparent longer persistence of the CAR-T cell product translates to more durable responses.

\section{P-BCMA-101}

P-BCMA-101 utilizes an anti-BCMA Centyrin ${ }^{\mathrm{TM}}$ (Aro Biotherapeutics Company, Philadelphia, PA, USA) fused to a CD3६/4-1BB signaling domain with a larger range of binding affinities, and is smaller, more stable and potentially less immunogenic. The piggyBac ${ }^{\mathrm{TM}}$ (PB) DNA modification system, as opposed to a viral vector, requires only plasmid DNA and mRNA producing an increased memory-like phenotype population. The higher cargo capacity allows for the incorporation of other genes, a safety switch that allows for rapid depletion of product in vivo if indicated by adverse events, and a selection gene that allows for enrichment of CAR+ cells. ${ }^{40} \mathrm{~A}$ phase I dose escalation study from 0.75-15 x 106 P-BCMA-101 CAR-T cells/kg was conducted in patients with RRMM; only one patient experienced low-grade CRS in the first cohort. Longer-term outcomes are still pending. ${ }^{41}$ The PRIME phase II study of the P-BMCA-101 product evaluates outpatient infusion as well as novel design features. ${ }^{42}$ A new manufacturing process utilizing a NanoplasmidTM improved ORR at a dose of $0.75 \times 10^{6}$ from $66.7 \%$ down to $50 \%$ and will be utilized as the manufacturing process moving forward. ${ }^{43}$

\section{СТ053}

CT053 is a second-generation CAR utilizing a fully human BCMA-specific single chain fragment variant $(25 \mathrm{C} 2)$ with high binding affinity. The CAR was initially studied in a single arm phase I investigator-initiated program in Eastern China. A median 17.7-month follow up reported on 21 patients receiving the $1.5 \times 10^{8} / \mathrm{kg}$ dose level after standard lymphodepleting chemotherapy (LDC). CR was low grade at $62.5 \%$ with a median onset of 3 days. Response showed an ORR of $87.5 \%$ with an impressive $79.3 \%$ CR rate. Median PFS was 18.8 months with improved DOR in patients without extramedullary disease (21.8 months versus 10.3 months). Persistence of the CAR-T cells was noted up to 161 days, and persistence correlated with response. ${ }^{44}$ To confirm these results, a phase Ib/II study, LUMMICAR-2, is enrolling subjects who have received three or more prior lines of therapy including a PI, an IMiD, and an anti-CD38 antibody. ${ }^{45}$ Patients receive standard LDC with a single infusion of CT053 with a dose level 0 targeted at $1.5-1.8 \times 10^{8} / \mathrm{kg}(n=8)$ and an escalated dose level 1 targeted at 2.5-3 $\times 10^{8} / \mathrm{kg}(n=6)$. The manufacturing time was $8-10$ days. Ten patients were 
evaluable at a median follow up of 4.5 months. CR rates were low grade $77 \%$ and $83 \%$, with a median onset of 2 days and 4 days, at dose level 0 and dose level 1, respectively. An ORR of $94 \%$ with CAR-T cell expansion and persistence was noted for up to 6 months. The DOR and depth of response continues to deepen over time. ${ }^{45}$

\section{Future of CAR-T cells for multiple myeloma Incorporating CARs into earlier lines of therapy}

While the second wave of CAR-T cell therapy in RRMM has focused mainly on product persistence to address the durability of response, future directions are multifaceted (Table 3). Both ide-cel and cilta-cel are in the process of conducting studies in the earlier relapsed setting of 1-3 prior treatments. Patients with high-risk MM are an attractive population in which to offer earlier CAR T-cell therapy given their significantly lower ORR, and shorter median PS and OS compared to standard-risk patients.

\section{Bispecific CARs, CARs in tandem, novel targets}

$\mathrm{MM}$ is a disease of high clonal competition and, as previously mentioned, antigen loss in a forthcoming clone can account for BCMA CAR-T cell relapse. Therefore, another approach to increase ORR and PFS in CAR-T cell therapy is dual antigen targeting to mitigate antigen loss with the treatment of different clones. Partnering BCMA targeting with anti-CD19 can trigger the elimination of malignant cells as CD19 is expressed on both myeloma cells and their progenitors. GC012F-a first-in-human BCMA/CD19 dual CAR - was constructed by linking BCMA and CD19 SCFV joined by a CD8 hinge, transmembrane domain, co-stimulatory domain, and CD3 $\zeta$. Patients have been enrolled at three dose levels with a primary end point of doselimiting toxicity of GC012F, and secondary endpoints of MRD at the 3- and 6-month infusion, ORR, PFS, OS, and DOR. Sixteen patients have been enrolled, including six at the third dose level of $3 \times 10^{5} / \mathrm{kg}$ cells. The ORR of the study is $93.8 \%$ at 6 months, MRD-negative of $100 \%$ at 3 and 6 months at dose level 3, and no dose-limiting toxicity (DLT). Low CR grades are present at $87.5 \%$ with a median onset of 6 days. ${ }^{46}$

Other researchers have looked at using unique antigen targeting CAR-T cellss in tandem dosing to address higher-risk populations. A single-center, single-arm, phase $\|$, feasibility study enrolled 21 patients who received lymphodepleting chemotherapy followed by humanized anti-CD19 CAR-T cells on day 0 , and split dose murine anti-BCMA CAR-T cells on days 1 and 2 . At a median follow up of 179 days, 20 of the 21 patients showed a response to treatment. ${ }^{47}$ Subsequently, a combined infusion has been escalated to 10 patients with R-ISS III disease with tandem autologous transplantation and combined infusion of CAR-T-19 and CART-BCMA cells post-transplant as consolidation treatment. CR occurred in all patients, limited to grade 1 and 2 , and $100 \%$ of patients achieved VGRP at 100 days post autologous stem cell transplant (ASCT). ${ }^{48}$ Another trial is treating high-risk patients to consolidate their first or second line of therapy with CART-BCMA plus or minus huCART19. ${ }^{21}$ Non-BCMA targets-other than CD19-are also being investigated including products directed at CD38, CD138, CS1/SLAMF7, APRIL, and GPRC5D (Table 3). ${ }^{49-54}$

\section{Gamma secretase inhibitors}

A novel CAR product seeks to prevent MM relapse post CAR-T cell infusion utilizing GSI drug infusions with an anti-BCMA CAR. GSIs increase BCMA surface density and decrease SBMCA, thus theoretically increasing the efficacy of the anti-BMCA CARs. Patients received GSI
Table 3: Future directions of cellular therapy research in the treatment of multiple myeloma

\begin{tabular}{|l|l|}
\hline Timing & $\begin{array}{l}\text { Earlier lines of therapy } \\
\text { Front-line in high-risk disease or as consolidation/ } \\
\text { maintenance }\end{array}$ \\
\hline Novel constructs & $\begin{array}{l}\text { Bispecific CARs, suicide genes, safety signals, PD-1 } \\
\text { knockouts }\end{array}$ \\
\hline Combinations & $\begin{array}{l}\text { GSI, IMiDs, checkpoint blockade, vaccines, monoclonal } \\
\text { antibodies, cytokines, multiple(tandem) CARs }\end{array}$ \\
\hline Alternate targets & CD38, CD138, CS1/SLAMF7, APRIL, GPRC5D \\
\hline Allogeneic donor & "Universal" or "off-the-shelf" CAR-T cells \\
\hline Alternate cells & Natural killer cells \\
\hline
\end{tabular}

$C A R=$ chimeric antigen receptor; GSI = gamma secretase inhibitor; IMiDs = immunomodulating drugs; $P D-1=$ programmed cell death protein-1.

(JSMD194) monotherapy administered every 48 hours over 5 days multiplied by three doses prior to Iymphodepleting chemotherapy, and then infusion of a fully humanized BCMA CAR in combination with JSMD194 dosed three times weekly for 3 weeks, starting on the day of CAR infusion. The run-in dosing of JSMD194 increased plasma cell BCMA expression from $75 \%$ to $99 \%$, and soluble BCMA decreased two-fold. BCMA antigen binding capacity increased to a median of 20 -fold and best overall response rate was $100 \%$ in six evaluable patients. ${ }^{.1}$

\section{Allogeneic CAR-T cells}

Until now, the majority of CAR-T cell products have been autologous. There are several issues that arise as a result, including: insufficient collection of $T$ cells; failure of engineering BCMA CAR-T cells; suboptimal T-cell substrate; and a prolonged time period between collection and infusion of cells. Allogeneic CAR-T cells may be able to overcome these issues by providing readily available off-the-shelf CAR-T cells without the need for prior autologous collection. New issues introduced by the use of an allogeneic product is the potential for graft-versus-host disease (GVHD), and lack of persistence. There are several products currently in development and in early clinical trials. First studies are now open to enrollment evaluating the safety of allogeneic CAR-T cell products targeting myeloma cells. UCARTCS1 targets the antigen CS1, whereas the products BCMA-UCART, ALLO-715, CTX120, and PBCAR269A are all allogeneic BCMA CAR-T cell products that are currently being tested in phase I trials. ${ }^{49,50,55-57}$ Anti-BCMA ALLO-715 is an off-the-shelf CAR-T cell with 4-1BB co-stimulatory and CD3- $\zeta$ activation domains. The T-cell receptor alpha constant gene is disrupted to decrease GVHD risk, and the CD52 gene is disrupted to use ALLO-647 (an anti-CD52 monoclonal antibody) for selective and prolonged host lymphodepletion. The primary endpoint was tolerability with 31 patients with an average of five prior lines of therapy with all patients refractory to the last line of therapy; 94\% penta refractory patients showed low CR rates (45\%). Response and durability of the product were dose dependent, and the third dose level $\left(3.2 \times 10^{6}\right.$ cells) the ORR was $60 \%$ with the VGPR at $40 \%$ or better. ${ }^{58}$

\section{Natural killer CAR T cells}

Natural killer (NK) cells can identify and kill malignant cells in the absence of antibodies and major histocompatibility complex molecules. CAR-NK cells are commonly engineered from donated umbilical cord blood NK cell lines, such as NK-92 or induced pluripotent stem cells. ${ }^{59}$ 
One of the possible concerns is the short in vivo persistence, though it is unclear if persistence of a CAR-T cell product is really necessary to induce a sufficient response as seen in the CARTITUDE-1 study (ClinicalTrials.gov Identifier: NCT03548207), where loss of persistence of CAR-T cells did not correlate with lack of response. Thus, it is quite possible that a brief and rapid onset of killing might be sufficient to induce a durable response. Compared to CAR-T cell therapy, the potential benefits of CAR-NK cell therapy include "off-the-shelf" availability, and a potentially low toxicity profile. Therefore, it might be advantageous to perform repeated and frequent dosing with these cell products similar to bispecific antibodies. ${ }^{60}$ Preclinical data show that genetic modification of NK-92Ml cells with an anti-CD138 CAR enhances the cytotoxicity and potentiates the anti-myeloma effect with NK cell-based therapy. Both NKG2D-CAR NK cells and BCMA-CAR NK cells also appear to be equally efficient at eradicating MM cells. ${ }^{61}$ A phase I CAR-NK cell trial targeting BCMA for relapsed/refractory MM using adoptive BCMA CAR-NK 92 product is now open for enrollment. ${ }^{62}$

\section{Conclusion}

CAR-T cell therapy in the vulnerable RRMM patient population is showing unprecedented responses with single treatments of a biological agent as opposed to continuous therapy. Future directions are multifocal with increasing the quality of CAR products in terms of T-cell persistence with memory-like phenotypes, optimum CD4:CD8 ratios, and multi-antigen products, as well as timing of therapy in the treatment sequence. As patients progress on various treatments, they decrease their native T-cell populations, and have suboptimal T-cell input product for CAR-T cell production. Off-the-shelf CAR-T cell products from optimal T cell donors would avoid the problem of suboptimal autologous $T$ cell collection and obviate the delays in CAR-T cell production as off-the-shelf cellular products are readily available. More provocatively, placing CAR-T cell therapy earlier in the sequence of treatment takes advantage of a more robust host $\mathrm{T}$ cell population for CAR-T cell production, and adds the possibility of potential cure. Additionally, future trials seek to increase CAR-T cell treatment efficacy with modifications such as bispecific targeting or novel combinations with IMIDS, monoclonal antibodies, and other anti-MM drugs. Moving CARs to the outpatient setting is also part of the next frontier of cellular therapy, demanding outpatient management and prevention of CRS. Despite advances in treatment, there are still socioeconomic barriers to treatment for advanced therapies for RRMM. Future treatment will depend not only on the CAR-T cell product manufacturing success and treatment response, but also on patient access. $\square$
1. American Cancer Society. Cancer Facts and Figures, 2020. Available at: www.cancer.org/research/cancer-facts-statistics/ all-cancer-facts-figures/cancer-facts-figures-2020.html (accessed January 25,2021 .

2. Rajkumar SV. Myeloma today: Disease definitions and treatment advances. Am J Hematol. 2016:91:90.

3. Kumar SK, Dispenzieri A, Lacy MQ, et al. Continued improvement in survival in multiple myeloma: changes in early mortality and outcomes in older patients. Leukemia. 2014;28:1122-8.

4. Cornell RF, AA Kassim. Evolving paradigms in the treatment of relapsed/refractory multiple myeloma: increased options and increased complexity. Bone Marrow Transplant. 2016;51:479-91.

5. Sadelain M, Brentjens R, Riviere I. The basic principles of chimeric antigen receptor design. Cancer Discov. 2013:3:388-98.

6. van der Stegen SJ, Hamieh M, Sadelain M. The pharmacology of second-generation chimeric antigen receptors. Nat Rev Drug Discov. 2015;14:499-509.

7. Kochenderfer JN, Yu Z, Frasheri D, et al. Adoptive transfer of syngeneic $T$ cells transduced with a chimeric antigen recepto that recognizes murine CD19 can eradicate lymphoma and normal B cells. Blood. 2010:116:3875-86.

8. Maude SL, Laetsch TW, Buechner J, et al. Tisagenlecleucel in children and young adults with B-cell lymphoblastic leukemia. N Engl J Med. 2018;378:439-48.

9. Kochenderfer JN, Sommerville RPT, Lu T, et al. Lymphoma remissions caused by anti-CD19 chimeric antigen receptor $T$ cells are associated with high serum interleukin-15 levels. I Clin Oncol. 2017:35:1803-13.

10. Neelapu SS, Locke FL, Bartlett NL, et al. Axicabtagene ciloleucel CAR T-cell therapy in refractory large B-cell lymphoma. N Engl J Med. 2017;377:2531-44.

11. Garfall AL, Maus MV, Hwang W-T, et al. Chimeric antigen recepto T cells against CD19 for multiple myeloma. N Eng/ I Med 2015;373:1040-7

12. Cohen AD, Garfall AL, Stadtmauer EA, et al. Safety and efficacy of B-cell maturation antigen (BCMA)-specific chimeric antigen receptor T cells (CART-BCMA) with cyclophosphamide conditioning for refractory multiple myeloma (MM). Blood. 2017;130(Suppl. 1):505.

13. Carpenter RO, Evbuomwan MO, Pittaluga S, et al. B-cell maturation antigen is a promising target for adoptive T-cell therapy of multiple myeloma. Clin Cancer Res. 2013;19:2048-60.

14. Tai YT, Acharya C, An G, et al. APRIL and BCMA promote human multiple myeloma growth and immunosuppression in the bone marrow microenvironment. Blood. 2016;127:3225-36.

15. Lee L, Bounds D, Paterson J, et al. Evaluation of B cell maturation antigen as a target for antibody drug conjugate mediated cytotoxicity in multiple myeloma. Br J Haematol. 2016;174:911-22.

16. Carpenter RO, Evbuomwan MO, Pittaluga S, et al. B-cell maturation antigen is a promising target for genetically-modified T-cell therapy of multiple myeloma. Blood. 2012;120:937.

17. Ali SA, Shi V, Maric I, et al. T cells expressing an anti-B-cell. maturation antigen chimeric antigen receptor cause remissions of multiple myeloma. Blood 2016:128:1688-700.

18. Brudno JN, Maric I, Hartman SD, et al. T cells genetically modified to express an anti-b-cell maturation antigen chimeric antigen receptor cause remissions of poor-prognosis relapsed multiple myeloma. J Clin Oncol. 2018;36:2267-80

19. Raje N, Berjeda J, Lin Y, et al. Anti-BCMA CAR T-cell therapy bb2121 in relapsed or refractory multiple myeloma. N Eng/ J Med. 2019;380:1726-37.

20. Munshi NC, Anderson Jr LD, Shah N, et al. Idecabtagene vicleuce (ide-cel; bb2121), a BCMA-targeted CAR T-cell therapy, in patients with relapsed and refractory multiple myeloma (RRMM): Initial KarMMa results. J Clin Oncol. 2020;38:(Suppl.):8503.

21. Lin Y, Raje NS, Berdeja JG, et al. Idecabtagene Vicleucel (ide-cel, bb2121), a BCMA-Directed CAR T Cell Therapy, in Patients With Relapsed and Refractory Multiple Myeloma: Updated Results From Phase I CRB-401 Study. Presented at: 62nd American Society of Hematology Annual Meeting and Exposition, December 5, 2020. Abstr 131

22. Zhao W-H, Liu J, Wang B-Y, et al. A phase 1, open-label study of LCAR-B38M, a chimeric antigen receptor T cell therapy directed against B cell maturation antigen, in patients with relapsed or refractory multiple myeloma. J Hematol Oncol. 2018;11:141.

23. Wang B-Y, Zhao W-H, Liu J, et al. Long-term follow-up of a phase 1, first-in-human open-label study of LCAR-B38M, a structurally differentiated chimeric antigen receptor T (CAR-T) cell therapy targeting b-cell maturation antigen (BCMA), in patients (pts) with relapsed/refractory multiple myeloma (RRMM). Blood. 2019;134(Suppl. 1):579.

24. Berdeja JG, Madduri D, Usmani SZ, et al. Update of CARTITUDE-1: A phase Ib/II study of JNJ-4528, a B-cell maturation antigen (BCMA)-directed CAR-T-cell therapy, in relapsed/refractory multiple myeloma. J Clin Oncol. 2020;38:15(Suppl.):8505.

25. Zudaire E, Madduri D, Usmani SZ, et al Translational analysis from CARTITUDE-1, an ongoing phase 1b/2 study of JNJ-4528 BCMAtargeted CAR-T cell therapy in relapsed and/or refractory multiple myeloma (R/R MM), indicates preferential expansion of CD8+ T cell central memory cell subset. Blood. 2019;134(Suppl.1):928.

26. Madduri D, Berdeja JG, Usmani SZ, et al. CARTITUDE-1: Phase Ib/2 Study of Ciltacabtagene Autoleucel, A B-Cell Maturation AntigenDirected Chimeric Antigen Receptor T Cell Therapy, in Relapsed/ Refractory Multiple Myeloma. Presented at: 62nd American Society of Hematology Annual Meeting and Exposition, December 5,2020. Abstr 177

27. Ma T, Shi J, Liu H. Chimeric antigen receptor T cell targeting B cell maturation antigen immunotherapy is promising for multiple myeloma. Ann Hematol. 2019;98:813-22.

28. Cohen AD, Garfall AL, Dogan A, et al. Serial treatment of relapsed refractory multiple myeloma with different BCMA-targeting therapies. Blood Adv. 2019;3:2487-90.

29. Xu J, Chen L-J, Yang S-S, et al. Exploratory trial of a biepitopic CAR T-targeting B cell maturation antigen in relapsed/refractory multiple myeloma. Proc Natl A cad Sci U S A. 2019:116:9543-51.

30. Cohen AD, Garfall AL, Stadtmauer EA, et al. B cell maturation antigen-specific CAR T cells are clinically active in multiple myeloma. J Clin Invest. 2019;129:2210-21.

31. Friedman KM, Garrett TE, Evans JW, et al. Effective targeting of multiple B-cell maturation antigen-expressing hematological malignances by anti-B-cell maturation antigen chimeric antigen receptor T cells. Hum Gene Ther. 2018;29:585-601.

32. Wherry EJ.T cell exhaustion. Nat Immunol. 2011:12:492-9.

33. Jensen MC, Riddell SR. Designing chimeric antigen receptors to effectively and safely target tumors. Curr Opin Immunol. 2015;33:9-15.

34. Lin Q, Zhao J, Song Y, et al. Recent updates on CAR T clinical trials for multiple myeloma. Mol Cancer. 2019;18:154.
35. Mailankody S, Jakubowiak AJ, Htut M, et al. Orvacabtagene autoleucel (orva-cel), a B-cell maturation antigen (BCMA)-directed CART cell therapy for patients (pts) with relapsed/refractory multiple myeloma (RRMM): update of the phase 1/2 EVOLVE study (NCT03430011). J Clin Oncol. 2020:38(Suppl.): abstr 8504.

36. Perkins MR, Grande S, Hamel A, et al. Manufacturing an enhanced CAR T cell product by inhibition of the PI3K/Akt pathway during T cell expansion results in improved in vivo efficacy of anti-BCMA CAR T cells. Blood. 2015;126:1893.

37. Shah N, Alsina M, Siegel DS, et al. Initial results from a phase 1 clinical study of bb21217, a next-generation anti Bcma CART therapy. Blood. 2018:132(Suppl. 1):488.

38. Berdeja JG, Alsina M, Shah ND, et al. Updated results from an ongoing phase 1 clinical study of bb21217 anti-Bcma CAR T cell therapy. Blood. 2019;134(Suppl. 1):927.

39. Alsina M, Shah N, Raje NS, et al. Updated Results from the Phase 1 CRB-402 Study of Anti-Bcma CAR-T Cell Therapy Bb21217 in Patients With Relapsed and Refractory Multiple Myeloma: Correlation of Expansion and Duration of Response With T Cell Phenotypes. Presented at: 62nd American Society of Hematology Annual Meeting and Exposition December 5, 2020. Abstr 130

40. Barnett BE, Hermanson DL, Smith JB, et al. piggyBac TM-produced CAR-T cells exhibit stem-cell memory phenotype. Blood. 2016;128:2167.

41. Gregory T, Cohen $A D$, Costello $C L$, et al. Efficacy and safety of P-Bcma-101 CAR-T cells in patients with relapsed/refractory $(r / r)$ multiple myeloma (MM). Blood. 2018;132(Suppl. 1):1012.

42. Costello CL, Gregory TK, Ali SA, et al. Phase 2 study of the response and safety of P-Bcma-101 CAR-T cells in patients with relapsed/refractory ( $r / r$ ) multiple myeloma (MM) (PRIME). Blood. 2019;134(Suppl.1):3184.

43. Costello C, et al. Phase $1 / 2$ Study of the Safety and Response of P-BCMA-101 CAR-T Cells in Patients with Relapsed/Refractory Multiple Myeloma (PRIME) with Novel Therapeutic Strategies. Presented at: 62nd American Society of Hematology Annual Meeting and Exposition, December 5, 2020. Abstr 134.

44. Hao S, Jin J, Jiang S, et al. Two-Year Follow-up of InvestigatorInitiated Phase I Trials of the Safety and Efficacy of Fully Human B-Cell Maturation Antigen-Specific CAR T cells (CT053) in Patients with Relapsed/Refractory Multiple Myeloma. Presented at: 62nd American Society of Hematology Annual Meeting and Exposition, December 5, 2020. Abstr 132

45. Kumar S, Baz RC, Orlowski RZ, et al. Lummicar-2: A Phase 1/2 Study of Fully Human B-Cell Maturation Antigen-Specific CAR T cells (CT053) in Patients with Relapsed and/or Refractory Multiple Myeloma. Presented at: 62nd American Society of Hematology Annual Meeting and Exposition, December 5, 2020. Abstr 133 .

46. Jiang H, Dong B, Gao L, et al. Clinical Results of A Multicenter Study of the First-In-Human Dual BCMA and CD19 Targeted Nove Platform FAST CAR-T Cell Therapy for Patients With Relapsed/ Refractory Multiple Myeloma. Presented at: 62nd American Society of Hematology Annual Meeting and Exposition, December 5, 2020. Abstr 178

47. Yan Z, Cao J, Cheng $\mathrm{H}$, et al. A combination of humanised antiCD19 and anti-BCMA CAR T cells in patients with relapsed or refractory multiple myeloma: a single-arm, phase 2 trial. Lancet Haematol. 2019;6:e521-9.

48. Shi X, Yan L, Shang JJ, et al. Tandom autologous transplantation and combined infusion of CD19 and Bcma-specific chimeric 
antigen receptor T cells for high risk MM: initial safety and efficacy report from a clinical pilot study. Blood. 2018;132(Suppl.1):1009.

49. ClinicalTrials.gov. Study Evaluating Safety and Efficacy of UCART Targeting CS1 in Patients With Relapsed/Refractory Multiple Myeloma (MELANI-01). ClinicalTrials.gov Identifier: NCT04142619. https://clinicaltrials.gov/ct2/show/NCT04142619 (accessed February 3, 2021)

50. ClinicalTrials.Gov. Efficacy and Safety Evaluation of BCMA-UCART. ClinicalTrials.gov Identifier: NCT03752541. Available at: https:// clinicaltrials.gov/ct2/show/NCT03752541 (accessed February 3, 2021).

51. Cowan AJ, Pont M, Sather BD, et al. Efficacy and safety of fully human BCMA CAR T cells in combination with a gamma secretase inhibitor to increase BCMA surface expression in patients with relapsed or refractory multiple myeloma. Blood. 2019:134(Suppl. 1):204

52. Li C, Mei H, Hu Y, et al. A bispecific CAR-T cell therapy targeting $\mathrm{BCma}$ and $\mathrm{CD} 38$ for relapsed/refractory multiple myeloma: updated results from a phase 1 dose-climbing trial. Blood. 2019;134(Suppl.1):930
53. Popat R, Zweegman S, Cavet J, et al. Phase 1 first-in-human study of AUTO2, the first chimeric antigen receptor (CAR) T cell targetin APRIL for patients with relapsed/refractory multiple myeloma (RRMM). Blood. 2019;134(Suppl.1):3112.

54. Smith EL, Harrington $\mathrm{K}$, Staehr $\mathrm{M}$, et al. GPRC5D is a target for the immunotherapy of multiple myeloma with rationally designed CAR T cells. Sci Trans/ Med. 2019;11:eaau7746.

55. ClinicalTrials.gov. Safety and Efficacy of ALLO-715 BCMA Allogenic CAR T Cells in in Adults With Relapsed or Refractory Multiple Myeloma (UNIVERSAL). ClinicalTrials.gov Identifier: NCT04093596.

56. ClinicalTrials.gov. A Safety and Efficacy Study Evaluating CTX120 in Subjects With Relapsed or Refractory Multiple Myeloma. ClinicalTrials. gov Identifier: NCT04244656. Available at: https://clinicaltrials.gov/ ct2/show/NCT04093596 (accessed February 3, 2021).

57. ClinicalTrials.gov. A Dose-escalation Study to Evaluate the Safety and Clinical Activity of PBCAR269A in Study Participants With Relapsed/Refractory Multiple Myeloma. ClinicalTrials.gov Identifie NCT04171843. Available at: https://clinicaltrials.gov/ct2/show/ NCT04171843 (accessed February 3, 2021).
58. Mailankody S, Matous JV, Liedtke M, et al. Universal: An Allogeneic First-in-Human Study of the Anti-BCMA ALLO-715 and the Anti-CD52 ALLO-647 in Relapsed/Refractory Multiple Myeloma. Presented at: 62nd American Society of Hematology Annual Meeting and Exposition, December 5, 2020. Abstr 129.

59. Rezvani K. Adoptive cell therapy using engineered natural killer cells. Bone Marrow Transplant. 2019;54(Suppl.2):785-8.

60. Rezvani $K$, Rouce R, Liu E, et al. Engineering natural killer cells for cancer immunotherapy. Mol Ther. 2017;25:1769-81.

61. Maroto-Martín, E., Encinas J, Garcia-Ortíz A, et al. NKG2D and BCMA-CAR NK cells efficiently eliminate multiple myeloma cells. A comprehensive comparison between two clinically relevant CARs. Presented at: 24th European Hematology Association (EHA) Congress, June 15, 2019. Poster. PS1209.

62. ClinicalTrials.gov. Clinical Research of Adoptive BCMA CAR-NK Cells on Relapse/Refractory MM. ClinicalTrials. gov Identifier: NCT03940833. Available at: https://www.clinicaltrials.gov/ct2/ show/NCT03940833 (accessed February 3, 2021). 